\title{
Tonic-Clonic Seizure following Cytoreductive Surgery with Intraperitoneal Oxaliplatin: A Case Report and Review of the Literature
}

\author{
Jessica Sayuri Tsukamoto ${ }^{a}$ Marcos Belotto de Oliveira $^{b}$ \\ Renata D'Alpino Peixoto ${ }^{a}$ \\ Departments of ${ }^{a}$ Medical Oncology and ${ }^{b}$ Surgical Oncology, Hospital São José, São Paulo, \\ Brazil
}

\section{Key Words}

Peritoneal carcinomatosis · Cytoreductive surgery · Intraperitoneal chemotherapy ·

Oxaliplatin · Seizure

\begin{abstract}
Cytoreductive surgery (CRS) with hyperthermic intraperitoneal (IP) chemotherapy (HIPEC) is believed to improve outcomes in well-selected patients with peritoneal carcinomatosis. However, morbidity and mortality rates associated with this procedure are substantial. Here, we describe the case of a previously healthy young man who underwent CRS with hyperthermic IP oxaliplatin and developed one episode of tonic-clonic seizure on the second postoperative day.

\section{Introduction}

Dissemination and implantation of gastrointestinal malignancies throughout the peritoneal cavity result in peritoneal carcinomatosis (PC) [1], which is the second most frequent cause of death in colorectal cancer after metastatic disease to the liver [2]. In $25 \%$ of colorectal cancer cases, the peritoneal cavity is the only site of metastatic disease [3]. Despite recent advances in the development of systemic chemotherapeutic agents, PC is well known to portend a poor prognosis. Attempts have been made in the past decades to increase long-term survival in patients with PC by combining cytoreductive surgery (CRS) to remove visible

\section{KARGER}

Jessica Sayuri Tsukamoto

Department of Medical Oncology, Hospital São José

R. Martiniano de Carvalho, 965

São Paulo 01321-001 (Brazil)

E-Mail jessytsukamoto@ hotmail.com 
Tsukamoto et al.: Tonic-Clonic Seizure following Cytoreductive Surgery with Intraperitoneal Oxaliplatin: A Case Report and Review of the Literature

disease and hyperthermic intraperitoneal (IP) chemotherapy (HIPEC) to eradicate microscopic viable residual disease, with elevated and persistent drug concentration in the peritoneal cavity [4]. The peritoneal route of chemotherapy administration has the advantage of delivering the agent to the entire peritoneum after all adhesions have been lysed and before they have the opportunity to reform [1]. The peritoneal-plasma division concept allows a tenfold higher concentration of the chemotherapy to be reached in the abdominal cavity in direct contact with residual cancerous cells while minimal systemic absorption and adverse effects are expected. The addition of hyperthermia results in a potentiation of cytotoxicity, improving its capability of penetration into tumoral masses [4] and serving to rewarm the patient after a significant open procedure [1].

The ideal IP chemotherapeutic agent should be one that has maximal efficacy, offering optimal regional therapeutic benefits, while minimizing systemic toxicity [1]. Mitomycin C (MMC) has been the most frequently used IP chemotherapy for gastrointestinal malignancies. The high molecular weight of MMC limits its systemic absorption and toxicity after IP administration [5]. More recently, based on the efficacy of new systemic agents for patients with metastatic colorectal cancer, oxaliplatin and irinotecan have emerged as good options for peritoneal perfusion [6,7]. Although there are no randomized trials comparing MMC with oxaliplatin as IP chemotherapy, a retrospective study with 539 patients with PC associated with colorectal cancer showed that both agents achieve similar median overall survival (32.7 vs. 31.4 months; $p=0.925$ ) [8]. However, due to shortage of MMC in Brazil, we have been using oxaliplatin as the preferred agent.

Despite all those potential benefits associated with CRS with HIPEC, morbidity and mortality rates are substantial. Large series of patients undergoing CRS with HIPEC report mortality rates ranging from 1 to $8 \%$ [9-13]. In the only randomized study $(n=105)$ comparing systemic chemotherapy (fluorouracil-leucovorin) with or without palliative surgery with aggressive CRS with HIPEC, followed by the same systemic chemotherapy regime, the mortality rate in the CRS with HIPEC group was 8\% [3]. In addition, surgical morbidity, such as postoperative ileus, anastomotic fistula, and wound infection, as well as medical morbidity, including pharmacological toxicity, cytopenia, bone marrow aplasia, renal toxicity, and hydroelectrolytic disorders, are quite frequent following CRS with HIPEC [3, 9]. The most common reported complications include enteric fistula, intraabdominal abscess, pneumonia, small-bowel obstruction, pancreatitis, and neutropenia which often prolong the hospital stay.

To the best of our knowledge, there are no reported cases of seizure after CRS with HIPEC. Here, we describe the case of a previously healthy young man who underwent CRS with hyperthermic IP oxaliplatin and developed one episode of tonic-clonic seizure on the second postoperative day.

\section{Case}

A 26 year-old man presented in mid-2011 with increasing abdominal pain. In October 2011, he developed obstructive symptoms and underwent exploratory laparotomy. He was found to have a poorly differentiated adenocarcinoma of the transverse colon with multiple peritoneal implants. It was consistent with a pT4 pN2 (4/28) pM1 colonic adenocarcinoma, KRAS wild-type. From January to July 2012, he received 12 cycles of FOLFIRI (folinic acid, 5fluorouracil, and irinotecan) plus bevacizumab at another institution and achieved complete clinical response. At that time, it was decided to stop chemotherapy completely. In September 2013, he again developed abdominal pain. A PET-CT scan revealed lesions with in- 
Tsukamoto et al.: Tonic-Clonic Seizure following Cytoreductive Surgery with Intraperitoneal Oxaliplatin: A Case Report and Review of the Literature

creased FDG uptake in the peritoneum as well as in the colonic anastomosis. From June 2014 to June 2015, he received 24 cycles of FOLFIRI and cetuximab with initial response followed by stable disease. In September 2015, he was first seen at our institution and, during a multidisciplinary meeting, it was decided to pursue CRS with HIPEC (oxaliplatin $300 \mathrm{mg} / \mathrm{m}^{2}$ ), which was started on October 5,2015 . On the second postoperative day, the patient developed one episode of generalized tonic-clonic seizure which lasted for approximately 2 min. He had no history of previous seizures. A CT scan of the head showed no abnormalities (fig. 1), there were no electrolyte disturbances, and no evidence of infection. He was started on phenytoin to prevent new episodes of seizures during the postoperative period. On October 15 , due to no recurrence of seizures, the anticonvulsant was discontinued. He stayed at the ICU for only $72 \mathrm{~h}$ and had the first bowel movement 6 days after surgery. On the 7 th postoperative day, he developed fever with no identified origin and was started on piperacillintazobactam and vancomycin. At this time, the patient was also complaining of left scrotal pain and swelling. An ultrasound of the testis was performed on October 17 and showed normal testicles and increased volume of the left epididymis with heterogeneous echogenicity. In addition, analysis of the epididymal waveform revealed a low-resistance pattern suggestive of acute epididymitis. Despite all cultures having remained negative, it was decided to switch antibiotics to amikacin for 10 days and to doxycycline for 14 days and treat the symptoms as infectious epididymitis. The patient was discharged home on October 22, 2015, to complete antibiotics at the outpatient unit.

\section{Discussion}

A seizure is a transient epileptic event, indicating a disorder in brain activity [14]. Ten percent of adults experience a seizure some time during their life [15]. Each year, 300,000 people experience a first epileptic seizure; most of them are younger than 18 years old [15]. Seizures result from a shift in the normal balance of excitation and inhibition within the central nervous system as well as from abnormal brain function [16]. Studies indicate that 25$30 \%$ of first seizures are 'acute symptomatic' or 'provoked' by a brain insult or a metabolic/toxic disturbance of brain function. Provoking factors include fever, head injury, excessive alcohol intake, withdrawal from alcohol or drugs, hypoglycemia, electrolyte disturbance, brain infection, ischemic stroke, intracranial hemorrhage, and proconvulsive drugs (such as clozapine, maprotiline, tramadol, theophylline, and baclofen) [17].

There are several alterations in the physiology of patients during CRS and the administration of HIPEC. The body temperature increases significantly due to HIPEC, they experience transient hyperglycemia secondary both to stress from surgery and hyperthermia, as well as the presence of dextrose in the perfusate [1]. However, none of those abnormalities has ever been reported to cause seizures, as seen in our case. There are no published articles regarding neurological complications such as seizures in patients who were submitted to HIPEC with oxaliplatin. There is, however, one case report of a 37-year-old female patient with a previous history of seizure disorder who underwent CRS and HIPEC with MMC without intraoperative complications and developed signs and symptoms of cerebral edema $4 \mathrm{~h}$ after the operation was completed. She eventually died of cerebral edema, with no established etiology [14]. Despite a normal EEG, it was hypothesized that CRS with HIPEC could have caused unrecognized partial complex seizures leading to acute cerebral edema. Even though our patient had no history of seizure disorder, it is a possibility that the seizure threshold may have been lowered secondary to the stress of surgery and hyperthermia. 
Tsukamoto et al.: Tonic-Clonic Seizure following Cytoreductive Surgery with Intraperitoneal Oxaliplatin: A Case Report and Review of the Literature

We have also hypothesized that IP oxaliplatin could lead to tonic-clonic seizure. In fact, seizures are a rare side effect of intravenous oxaliplatin, which are usually associated with posterior reversible leukoencephalopathy syndrome (PRES). PRES is characterized by neurologic symptoms such as headache, altered mental status, visual disturbances, and seizures with typical lesions on neuroimaging shown as bilateral, subcortical, symmetric, and vasogenic edema [18]. However, our patient had no symptoms or radiologic findings of PRES. There is a published case of a previously healthy man with mixed adenoneuroendocrine carcinoma who had seizures after the third and fourth cycles of intravenous folinic acid, 5fluorouracil, and oxaliplatin (FOLFOX) with no signs of PRES. After the chemotherapy regimen was switched to FOLFIRI, he never had another event. In this case, the authors attributed the seizures to the intravenous oxaliplatin [19].

Our patient had no electrolyte abnormalities, no signs of infection, brain structural alteration, or other metabolic disorder that could possibly explain the single episode of seizure. Therefore, it may be possible that the seizure was caused either by the physiological modifications that occurred during HIPEC or by IP oxaliplatin. Because of the increasing use of CRS with HIPEC as an option to treat selected patients with PC, it is important to report those postoperative complications.

\section{Conclusion}

This article reports an unprecedented case of seizure in a previously healthy patient who underwent CRS with IP oxaliplatin due to metastatic colon adenocarcinoma. Despite this being a rare case, the recognition of this potential complication is important for physicians performing CRS and HIPEC.

\section{Statement of Ethics}

The corresponding author acknowledges that she is responsible for complying with ethical requirements and declares that the patient was correctly informed, and written informed consent was obtained; the confidentiality of the patient was strictly preserved. The patient was informed about the submission of the manuscript and will be acquainted when the article is published.

\section{Disclosure Statement}

The authors declare that they have nothing to disclose.

\section{References}

1 Ihemelandu CU, Shen P, Stewart JH, et al: Management of peritoneal carcinomatosis from colorectal cancer. Semin Oncol 2011;38:568-575.

2 Vaira M, Cioppa T, D'Amico S, et al: Treatment of peritoneal carcinomatosis from colonic cancer by cytoreduction, peritonectomy and hyperthermic intraperitoneal chemotherapy (HIPEC). Experience of ten years. In Vivo 2010;24:79-84.

-3 Verwaal VJ, Ruth SV, Bree E, et al: Randomized trial of cytoreduction and hyperthermic intraperitoneal chemotherapy versus systemic chemotherapy and palliative surgery in patients with peritoneal carcinomatosis of colorectal cancer. J Clin Oncol 2003;21:3737-3743. 


\section{Case Reports in Oncology}

\begin{tabular}{l} 
Case Rep Oncol 2016;9:89-94 \\
\hline DOI: $10.1159 / 000443827$ \\
\hline
\end{tabular}

Tsukamoto et al.: Tonic-Clonic Seizure following Cytoreductive Surgery with Intraperitoneal Oxaliplatin: A Case Report and Review of the Literature

4 Vaira M, Scuderi S, Costamagna D, et al: Cytoreductive surgery and intraperitoneal hyperthermic-antiblastic therapy (HAPP) in peritoneal carcinomatosis. Minerva Chir 2002;57:597-605.

5 Lambert LA, Armstrong TS, Lee JJ, et al: Incidence, risk factors, and impact of severe neutropenia after hyperthermic intraperitoneal mitomycin C. Ann Surg Oncol 2009;16:2181-2187.

-6 Glockzin G, Gerken M, Lang SA, et al: Oxaliplatin-based versus irinotecan-based hyperthermic intraperitoneal chemotherapy (HIPEC) in patients with peritoneal metastasis from appendiceal and colorectal cancer: a retrospective analysis. BMC Cancer 2014;14:807.

7 Elias D, Lefevre JH, Chevalier J, Brouquet A, et al: Complete cytoreductive surgery plus intraperitoneal chemohyperthermia with oxaliplatin for peritoneal carcinomatosis of colorectal origin. J Clin Oncol 2009;27:681-685.

8 Prada-Villaverde A, Esquivel J, Lowy AM, Markman M, et al: The American Society of Peritoneal Surface Malignancies evaluation of HIPEC with mitomycin C versus oxaliplatin in 539 patients with colon cancer undergoing a complete cytoreductive surgery. J Surg Oncol 2014;110:779-785.

-9 Stephens AD, Alderman R, Chang D, et al: Morbidity and mortality analysis of 200 treatments with cytoreductive surgery and hyperthermic intraoperative intraperitoneal chemotherapy using the coliseum technique. Ann Surg Oncol 1999;6:790-796.

$\checkmark 10$ Glehen 0, Osinsky D, Cotte E, et al: Intraperitoneal chemohyperthermia using a closed abdominal procedure and cytoreductive surgery for the treatment of peritoneal carcinomatosis: morbidity and mortality analysis of 216 consecutive procedures. Ann Surg Oncol 2003;10:863-869.

$\checkmark 11$ Kusamura S, Younan R, Baratti D, et al: Cytoreductive surgery followed by intraperitoneal hyperthermic perfusion. Analysis of morbidity and mortality in 209 peritoneal surface malignancies treated with closed abdomen technique. Cancer 2006;106:1144-1153.

12 Nadler A, McCart JA, Govindarajan A: Peritoneal carcinomatosis from colon cancer: a systematic review of the data for cytoreduction and intraperitoneal chemotherapy. Clin Colon Rectal Surg 2015;28:234-246.

13 Desantis M, Bernard JL, Casanova V, et al: Morbidity, mortality, and oncological outcomes of 401 consecutive cytoreductive procedures with hyperthermic intraperitoneal chemotherapy (HIPEC). Langenbecks Arch Surg 2015;400:37-48.

14 Nair RL, Tobias J, Stemmerman G, et al: Cerebral edema in a patient following cytoreductive surgery and hyperthermic intraoperative intraperitoneal chemoperfusion. World J Surg Oncol 2006;4:85.

15 Angus-Leppan H: First seizures in adults. BMJ 2014;348:g2470.

16 Epilepsy Foundation: Epilepsy and seizure statistics. www.epilepsyfoundation.org/about/statistics.cfm (accessed November 18, 2015).

$\checkmark 17$ Pohlmann-Eden B, Beghi E, Camfield C, et al: The first seizure and its management in adults and children. BMJ 2006;332:339.

18 Staykov D, Schwab S: Posterior reversible encephalopathy syndrome. J Intensive Care Med 2012;27:11-24.

19 Rahal AK, Truong PV, Kallail KJ: Oxaliplatin-induced tonic-clonic seizures. Case Rep Oncol Med 2015;2015:879217. 


\section{Case Reports in Oncology}

\begin{tabular}{l|l}
\hline Case Rep Oncol 2016;9:89-94 \\
\hline DOI: 10.1159/000443827 & $\begin{array}{l}\text { c } 2016 \text { The Author(s). Published by S. Karger AG, Basel } \\
\text { www.karger.com/cro }\end{array}$ \\
\hline
\end{tabular}
www.karger.com/cro

Tsukamoto et al.: Tonic-Clonic Seizure following Cytoreductive Surgery with Intraperitoneal Oxaliplatin: A Case Report and Review of the Literature

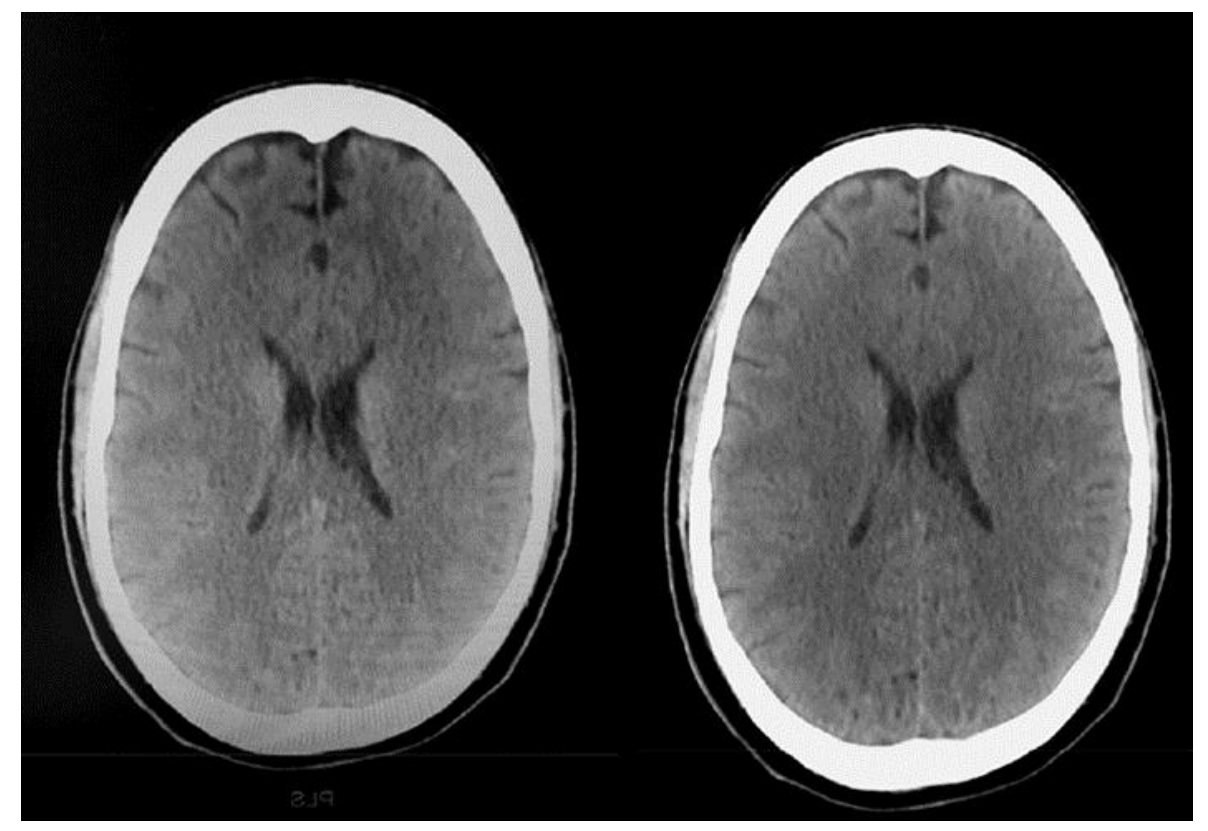

Fig. 1. Normal CT scan of the head. 